\title{
Percepción de docentes y supervisores sobre la implementación del modelo «1 a 1», One Laptop Per Child, en dos iniciativas públicas de Costa Rica
}

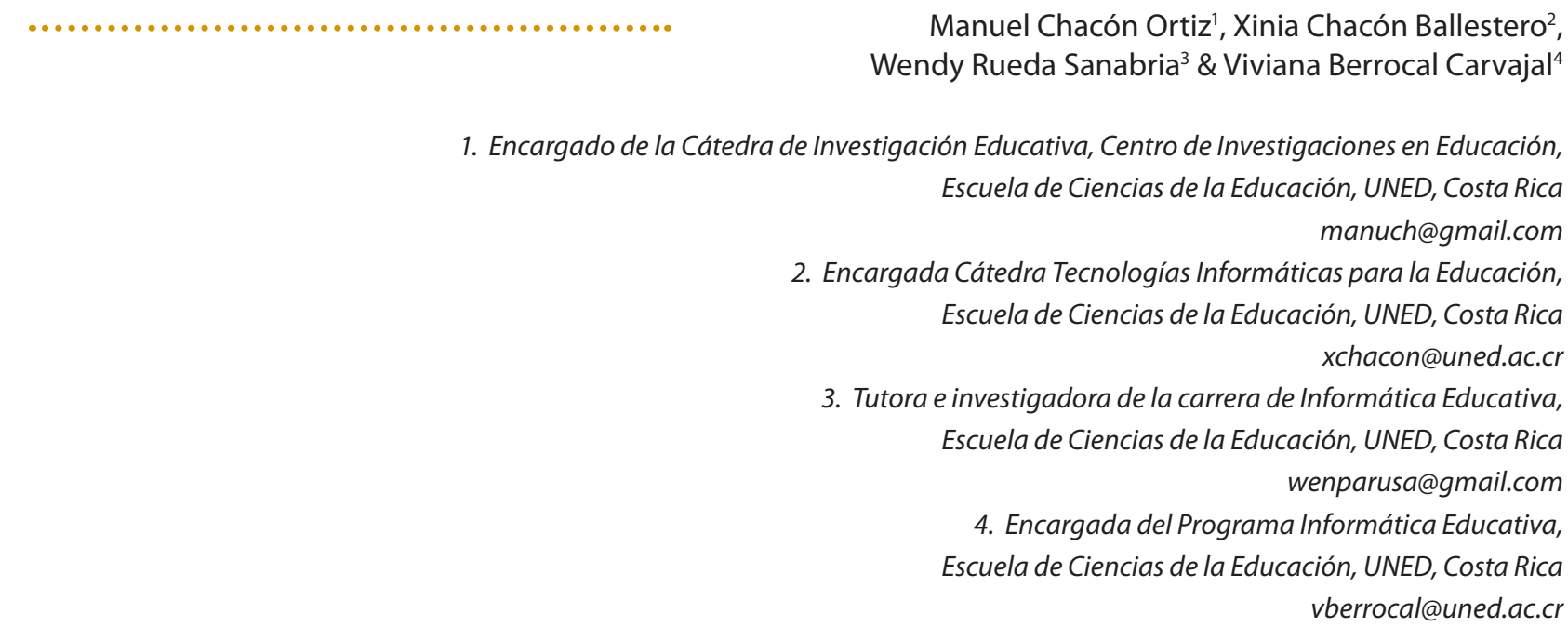

Recibido: 13 de octubre del 2017

Corregido: 16 de febrero del 2018

Aceptado: 21 de febrero del 2018

\begin{abstract}
Resumen
El presente estudio recoge las percepciones de docentes y asesores sobre el desarrollo de iniciativas sobre el modelo «1 a 1» en Costa Rica. El modelo One Laptop per Child es una iniciativa tecnológica implementada en varios países del mundo, consiste en dotar a estudiantes de una computadora portátil para el desarrollo de su práctica educativa dentro y fuera del aula. La metodología del trabajo es cuantitativa descriptiva. Se utiliza un cuestionario para recolectar información en dos iniciativas públicas. Los datos que se exponen corresponden a información recolectada con docentes y asesores, en total 47 personas, que participaron de estas iniciativas en el 2015. Se prestó asesoría al asignar los equipos necesarios y existe una actitud positiva desde los docentes que participaron del estudio. La principal limitación que se ha expuesto es el acceso a Internet en las instituciones, lo cual no permite aprovechar el recurso tecnológico en todo su potencial. Otra limitación es la falta de capacitación, la cual varía de acuerdo con cada iniciativa. Las iniciativas participantes del estudio han llevado a la práctica el modelo con un propósito claro de apoyo, cuya efectividad pedagógica y educativa aún es necesario profundizar en otras investigaciones.
\end{abstract}

Palabras Clave: Tecnología educativa, modelo «1 a 1», educación, aprendizaje, Educación General Básica, competencias docentes, Informática Educativa. 


\section{Abstract \\ Perception of teachers and supervisors on the implementation of the model «1 a 1», One Laptop Per Child, in two public initiatives of Costa Rica}

This paper presents the perception of teachers and advisers on the development of initiatives on the 1: 1 model in Costa Rica. The One Laptop per Child model is a technology initiative implemented in several countries around the world. It consists of equipping students with a laptop to develop their own educational practice in and outside the classroom. The methodology of this work is descriptive quantitative. A questionnaire is used to collect information in two public initiatives. The data presented correspond to information collected with teachers and advisors who participated, 46 people, in these initiatives in 2015. From the results, it is concluded that both initiatives have developed planned processes. Different types of equipment are granted. Advice was given when assigning the equipment and there is a positive attitude from the teachers who participated in this study. The main limitation is access to the Internet in the institutions, which does not allow taking advantage of the technological resource to its full potential. Another limitation is training, which varies according to each initiative. In conclusion, the initiatives participating in the study have implemented the model with a clear purpose of support, whose pedagogical and educational effectiveness is still necessary to deepen in future research.

Key words: Educational Technology, Model 1: 1, Education, Learning, General Basic Education, Teaching Competencies, Educational Informatics.

\section{INTRODUCCIÓN}

Hace unos años se consideraba como innovación contar con una computadora en cualquier centro educativo. Hoy día se promueve que cada estudiante cuente con su equipo propio.

En el año 2006, Nicholas Negroponte presenta el modelo de computadora portátil XO. La XO fue el modelo de bajo costo, creado con base en el sistema operativo Linux, cuyo valor aproximado en el momento de introducción rondaba entre los 100 y 200 dólares estadounidenses (Zucker y Light, 2009). La innovación se expone en el Foro Económico Mundial, con la idea de dotar a cada estudiante en países en vías de desarrollo de una computadora portátil. Esta iniciativa se le llamó entonces One Laptop Per Child (OLPC) (Diodato, 2007).

Algunas de las justificaciones que se han dado para la adopción del modelo por parte de distintos países han sido: promueve las destrezas tecnológicas en las y los estudiantes (Davies y West, 2014); reduce la brecha digital; promueve el acceso a mejores trabajos; accede a la población de escasos recursos, y forma a los estudiantes con destrezas sociales y tecnológicas acordes con las necesidades del siglo XXI.

El modelo propuesto por Negroponte no es la única versión del aprendizaje «1 a 1». Existen otras variantes de aprendizaje mediado por computadoras portátiles que no utiliza los sistemas XO. Sin embargo, esta iniciativa es la que ha tenido mayor impacto mediático.

\section{El contexto costarricense en relación con las computadoras portátiles}

A nivel público, en Costa Rica hasta el año 2015 las iniciativas «1 a 1» habían sido desarrolladas por tres instancias: Intel Educar, la Fundación Omar Dengo a través del programa MEP-PRONIE y la Fundación Quirós Tanzi. No obstante, es posible que otras instituciones educativas privadas implementaran algún tipo de modelo «1 a 1» en el uso de tecnología portátiles móviles. 
Es importante contextualizar las contradicciones tecnológicas que se dan en el país. Por una parte, en los últimos años se evidencia un crecimiento en el acceso y uso de tecnologías móviles por parte de la población. De acuerdo con información del censo en el año 2011, cuando se le pregunta a la población de 5 años y más por uso de tecnologías de información y comunicación en los últimos tres meses $72 \%$ manifiesta haber utilizado algún tipo de teléfono celular, en tanto $48 \%$ manifiesta haber utilizado una computadora y $45 \%$ haber empleado Internet (INEC, 2011). No obstante, persisten desigualdades significativas, pues tan sólo $32 \%$ de las viviendas contaba con una computadora de escritorio, y apenas $27 \%$ con una portátil (INEC, 2011). Si bien es cierto el abaratamiento de los costos de producción de computadoras ha facilitado el acceso a estas, para un sector de la población estas tecnologías siguen siendo un recurso distante.

En el contexto social donde las iniciativas «1 a 1» adquieren importancia, se convierten en una oportunidad de contrarrestar las desigualdades de acceso a poblaciones tecnológicamente excluidas. Además, el Tercer Informe del Estado de la Educación en Costa Rica (Proyecto Estado de la Nación, 2011) mostró un acceso desigual de las TIC en educación primaria, evidenciando un alto uso en las zonas urbanas y una exclusión en las zonas rurales.

\section{Experiencias internacionales bajo el modelo «1 a 1»}

El modelo «1 a 1» ha sido ampliamente investigado en los Estados Unidos y Europa. Según Area (2011) en el ámbito anglosajón (E.E. U.U., Reino Unido y Australia), desde hace algunos años se reporta sobre la evaluación e impacto del modelo. Esto se debe a que, mucho antes del modelo XO, los centros educativos de países desarrollados contaban con experiencias de dotar de portátiles para cada estudiante.

Las investigaciones originadas en Estados Unidos mostraron resultados importantes (Penuel, 2006): el haber brindado computadoras en las escuelas promovió el acceso ubicuo a la tecnología; se integraron en el aula como una herramienta productiva para tareas de clase; sin embargo, no es claro el efecto de estas tecnologías aplicadas a materias básicas, dado que pocas investigaciones habían reportado su uso en estas asignaturas; las experiencias más exitosas fueron aquellas que ofrecieron al docente la instrucción y los recursos curriculares para aprovecharlas como recursos tecnológicos; la actitud del docente frente a estas tecnologías marca una diferencia importante, y tienden a ser utilizadas más frecuentemente y de mejor forma por quienes las consideran como algo valioso.

Para el año 2007, casi 25\% de las escuelas estadounidenses habían implementado algún tipo de modelo «1 a 1», Holcomb (2009) advierte que la mayoría de las investigaciones no han mostrado un claro impacto. Agrega además (Steve Vosloo, 2014) que, muchos proyectos bajo este modelo en años recienten se han dado por razones políticas, económicas y presiones del mercado por colocar los equipos. No obstante, coincide en que el impacto académico aún no se ha podido comprobar. Al final concluye el autor, dos cosas deberían ser de primordial enfoque: no son las tecnologías, son las personas, y el modelo no es un fin en sí mismo, es un camino, sobre el cual se debe definir un claro objetivo.

Los estudios publicados en la literatura académica sobre impacto de las TIC en países en vías de desarroIlo no son abundantes (Kozma y Vota, 2014). Se realizan inversiones importantes, cuya evidencia de cambios en las poblaciones y escuelas donde se aplican no son certeros. Pero más importante, buena parte de los esfuerzos por los modelos «1 a 1» y XO han requerido una enorme inversión en infraestructura, indispensable para "arrancar" (cableado, internet, acondicionamiento de espacios), y que finalmente eran subutilizadas o estaban relegadas a prácticas educativas consideradas como tradicionales.

En el contexto latinoamericano, algunas instituciones como la Organización para la Cooperación y el Desarrollo Económicos (Valiente, 2010) y el Banco Interamericano de Desarrollo (Severin y Capota, 2011) desarrollaron estudios sobre las primeras experiencias bajo el modelo en países como Chile, Brasil y Uruguay. Se identificaron algunos elementos importantes en torno a estas iniciativas: la mayoría deseaba brindar herramientas básicas para el manejo de las TIC a los estudiantes con el fin de reducir la brecha 
digital; la mayor parte de las experiencias «1 a 1» en países en vías de desarrollo han sido a gran escala; en todas existe una intervención entre el sector público y privado; el soporte técnico es un aspecto fundamental; aunque ha habido poco conocimiento sobre la implementación, los beneficios académicos y educativos de estas iniciativas, la mayoría de las evaluaciones a la fecha, basadas únicamente en percepciones, han sido positivas (Valiente, 2010). No obstante, aún falta evidencia cualitativa y cuantitativa sobre el aprovechamiento de los recursos tecnológicos en los salones de clase.

Es importante resaltar algunas experiencias latinoamericanas significativas. El Ministerio de Educación y Cultura de Uruguay con el Plan Ceibal fue pionero en América Latina bajo el modelo «1 a 1». La iniciativa inicia en el 2007 con portátiles XO. La intención era disminuir la brecha digital y mejorar el proceso de enseñanza-aprendizaje para lograr una mayor alfabetización digital en los estudiantes. El principal logro ha sido abarcar toda la población de educación primaria en Uruguay (Edelman y Fernández, 2010).

Otras iniciativas han sido el proyecto Laboratorio Móvil Computacional (LMC) Modelo 1 a 1 Chile en el año 2009 (Claro, Nussbaum, López, y Díaz, 2013) y en Paraguay, la organización Educa desarrolló modelos «1 a 1» apoyados de entidades gubernamentales (Warschauer y Ames, 2010), iniciativa similar, pero a mayor escala, que llevó a cabo el Ministerio de Educación de la Ciudad de Buenos Aires con el Plan Integral de Educación Digital (Nugroho y Londsale, 2010).

La inclusión de la tecnología educativa ha sido puesta en práctica por empresas, gobiernos, y organizaciones no gubernamentales. Lo anterior ha llevado a muchos países a replantearse los modelos educativos para una transformación a nivel curricular y la construcción de nuevas prácticas pedagógicas, en las que la tecnología no es un complemento, sino parte de la actividad cotidiana del aula.

\section{Experiencias bajo el modelo «1 a 1» en Costa Rica}

Las primeras experiencias de aprendizaje móvil se dieron en el marco de la Fundación Omar Dengo. Primeramente, el proyecto Aprendizaje con Tecnologías Móviles en escuelas multigrado del año 2003. La experiencia inició con laboratorios mixtos de computadoras portátiles y estacionarias en el Tercer Ciclo de Enseñanza General Básica (Fundación Omar Dengo, s. f.). Así mismo, la fundación fue precursora al colaborar con experiencias en el modelo «1 a 1» con su proyecto en localidades como El Silencio, en Tilarán, acompañados del MIT (Massachusetts Institute of Technology). El proyecto inicia en el 2005 y se extiende por dos años como un plan piloto en escuelas unidocentes (Alvarado-Barrantes, 2007).

En el año 2008 se desarrolla el Programa de Investigación y Desarrollo en Ambientes de Aprendizaje con Tecnologías Móviles e inicia el proyecto Generación «1 a 1» utilizando ClassMate, en colaboración con la compañía Microsoft (Vacchieri, 2013). Asimismo, surge el proyecto Tecnologías Móviles en Educación en diez instituciones de educación primaria con ClassMate, auspiciado por la compañía INTEL y el MEP. Este fue, en su momento, uno de los proyectos más grandes en el país (MEP, 2014).

Para el año 2009 la Fundación Omar Dengo y el Ministerio de Educación llevan a cabo el proyecto Rem@: Redes Móviles para el Aprendizaje, proyecto de tecnologías móviles en liceos rurales tuvo como fin dotar a cada educador y estudiante del centro educativo de una computadora portátil para su utilización dentro y fuera de la institución (MEP, 2014). Las iniciativas se enmarcan en las gestiones del Programa Nacional de Informática Educativa MEP-FOD.

En el año 2013 se crean los proyectos Movilab Primaria y secundaria, iniciativas del PRONIE MEP-FOD, donde se dota a escuelas y colegios de computadoras portátiles, a los cuales se suma el proyecto de Tecnologías Móviles en Centros Educativos Indígenas del año 2013 (Fundación Omar Dengo, s. f.).

El Ministerio de Educación Pública (MEP) es otro actor clave en el proceso a través de la Dirección de Recursos Tecnológicos. Desde el año 2015, han implementado el Programa Nacional de Tecnologías Móviles cuyo objetivo es "contribuir al desarrollo de la educación costarricense, por medio de la inclusión 
de tecnologías digitales móviles en los procesos de enseñanza y aprendizaje, como apoyo al currículo nacional" (MEP, 2016, p. 5).

El programa citado abarca todos los niveles educativos, desde preescolar hasta secundaria, y se desarrolla también de la mano con las iniciativas de la FOD, y del proyecto Conectándonos de la Fundación Quirós Tanzi, el cual inicia en el año 2011, y ya para el año 2013 había dotado a 1500 estudiantes y más de 40 escuelas, capacitando a más de 260 docentes (Fundación Quirós Tanzi, 2013). El programa ha permitido que niños y docentes de escuelas que actualmente no cuentan con plataformas informáticas cuenten con una computadora personal, así como toda la estructura necesaria para la interconexión de los equipos. El convenio incluye mantenimiento y capacitación continua y, a diferencia de los otros proyectos, sí se enmarca en la propuesta OLPC original utilizando los sistemas XO (One Laptop per Child, 2011).

\section{Consideraciones sobre la implementación del modelo «1 a 1»}

Los modelos iniciales se basaron en computadoras portátiles regulares, con sistemas operativos Windows. El propósito principal de las iniciativas informáticas «1 a 1» es aumentar el acceso a la tecnología en las escuelas (Davies y West, 2014). Las características que sigue el modelo «1 a 1» en países en vías al desarrollo muestran connotación tecnológica, educativa, y social. Las iniciativas han sido parte de una política pública que busca reducir la brecha digital a partir de un cambio a nivel del currículo escolar (Kozma y Vota, 2014).

Originalmente, se trataba de que cada estudiante posea una computadora de escritorio en el aula que pueda utilizar como herramienta de apoyo para su aprendizaje (Penuel, 2006). Sin embargo, con el advenimiento de las tecnologías móviles, laptops y diferentes dispositivos portátiles el concepto ha evolucionado a una computadora portátil por estudiante. Actualmente en la región, los dispositivos más usados para las iniciativas uno a uno son las computadoras portátiles (Severin y Capota, 2011, p. 6). La meta es que cada estudiante tenga acceso a una computadora portátil para fines educativos.

El modelo más reconocido, en países en vías de desarrollo, es el que desarrolló el MIT: One Laptop Per Child, sus principios fueron creados por el proyecto OLPC con el fin de incentivar la autonomía e independencia del estudiante como un ser que se apropia de su aprendizaje, y es capaz de acceder a conocimientos en la escuela, pero también fuera de ella, en su casa, en su comunidad. Se pueden resumir estos principios de la siguiente forma (The OLPC Wiki, s. f.):

- Niño propietario: la computadora se le entrega al niño, él puede trasladarla de la escuela a su casa y convertirse en un agente de alfabetización computacional para su familia.

- Niños pequeños: las computadoras están destinadas a niños de edad escolar entre los seis y doce años por ser los períodos en que se desarrollan las diferentes habilidades cognoscitivas.

- Saturación: refiriéndose a la saturación digital, el proyecto busca que varias instituciones educativas de la comunidad cuenten con una computadora por niño.

- Conectividad: computadoras que están conectadas entre sí lo cual permite que los niños que las tienen están en constante comunicación.

- Código abierto-libre: no existe ninguna dependencia de software, o restricciones de redistribución.

La computadora XO es pequeña, se clasifica como una laptop, posee un hardware diseñado para permitir la larga duración de su batería, es portable, tiene comunicación Wifi, conexión a dispositivos USB y mouse. Cuenta con una memoria flash que le permite almacenar el sistema operativo y la información. Posee también una cámara de video, conexión para micrófono y parlantes. El sistema estaba basado en una licencia GNU con núcleo. Tiene un software de comunicaciones que le permite mantener la 
conectividad con otras máquinas XO (Ryan, 2014). El diseño de esta máquina permite y facilita el trabajo, portabilidad y durabilidad de los equipos.

Las computadoras XO trabajan diferentes lenguajes y herramientas de programación que grafican, ilustran y crean multimedia con la información. Lo anterior se lleva a cabo por medio de una plataforma de software libre basada en Linux Ilamada Sugar, que le ofrece al estudiante una serie de actividades de aprendizaje necesarias para el registro de la información obtenida, la colaboración y la expresión de ideas.

En el proyecto el rol del estudiante es el de una persona activa, capaz de construir y reconstruir sus propios conocimientos, trabaja de forma colaborativa con pares y comparte lo que produce. El educador participa como mediador de los procesos de aprendizaje, se constituye en agente facilitador de los conocimientos. En el modelo OPLC, la familia y la comunidad son agentes participantes en el proceso educativo mediante la comunicación entre los padres y el centro educativo, de manera que ambas esferas pueden brindar el apoyo necesario y aprender de la misma experiencia.

Además de lo anterior, otros elementos que caracterizan la implementación de los modelos «1 a 1» son la capacitación al personal docente (UNESCO, 2016), por las actividades de enseñanza y aprendizaje que les permite llevar a cabo actividades de forma ubicua, por medio de distintas herramientas digitales, y a través de diferentes software (Pachler, Bachmair, y Cook, 2010). Bajo estas premisas epistemológicas, el fin del estudio es conocer, desde la perspectiva docente, cómo se valora la implementación del modelo «1 a 1» en relación con la infraestructura, condiciones de los equipos, actividades y capacitación.

Ante el panorama descrito, se plantea la siguiente interrogante: ¿cuál es la percepción que de los profesores y asesores sobre la implementación de los proyectos educativos con tecnologías móviles bajo el modelo «1 a 1» en las cuáles han participado?

\section{METODOLOGÍA}

Tipo de estudio. El estudio se desarrolló bajo un paradigma naturalista descriptivo (Hernández Sampieri, Fernández Collado y Baptista Lucio, 2010). Con este fin se llevó a cabo una selección exploratoria de las tres principales iniciativas «1 a 1» existentes en el país (Flyvbjerg, 2011).

Población. Se tomaron dos casos de proyectos vigentes al momento de recolectar la información durante el año 2015 en instituciones públicas: los proyectos llevados a cabo por el PRONIE-FOD MEP, y el proyecto Conectándonos de la Fundación Quirós Tanzi. Con respecto al tercer caso, el proyecto INTEL Educar, una de las primeras experiencias que se llevó a cabo en el país, no fue posible de sistematizar desde un cuestionario la experiencia de los participantes debido a que el proyecto finalizó en el año 2011, aproximadamente, y no existía al momento de realizar la investigación una red de contactos para valorar la experiencia.

Muestra. Dada la selección intencional de las iniciativas, no se siguió una lógica de muestreo, pues responde a ciertas características que se ha propuesto el equipo de trabajo (Day-Ashley, 2012). La selección de la muestra de participantes, por la naturaleza del estudio, fue a conveniencia (Hewson, Vogel, y Laurent, 2016). Se convocó a los participantes por medio de las personas a cargo de la dirección de ambos proyectos en el país, quienes apoyaron la difusión del cuestionario por medio de correos o mensajes de texto.

Instrumento. Se trabajó por medio de un cuestionario con escalas con el fin de valorar la experiencia de los participantes de la investigación (Tymms, 2012). La selección de un cuestionario con escala responde a la posibilidad de recolectar y unificar algunos criterios que para el equipo de trabajo fueran 
importantes sobre la experiencia de modelos «1 a 1». El instrumento se sometió a criterio de expertos en informática educativa, y fue validado previo por las directoras de ambas iniciativas donde fue aplicado.

Limitaciones. Como parte de las limitaciones encontradas en la ejecución de la investigación y la recolección de información se encuentra no haber podido acceder a una mayor cantidad de sujetos de información. El envío del instrumento a través de listados propios de las instituciones contribuyó, pero no fue suficiente para obtener una mayor cantidad de respuestas.

TABLA 1

Variables contempladas en la investigación

\begin{tabular}{|c|c|c|}
\hline Variables & Definición conceptual & Definición operacional \\
\hline Institucionales & $\begin{array}{l}\text { Corresponde al tipo de institución y características de las } \\
\text { personas participantes del estudio }\end{array}$ & $\begin{array}{l}\text { Grado académico, sexo, } \\
\text { tipo de escuela o función, iniciativa en la } \\
\text { que participa }\end{array}$ \\
\hline $\begin{array}{l}\text { Condiciones e } \\
\text { infraestructura }\end{array}$ & $\begin{array}{l}\text { Corresponde con aspectos como equipo, conexión a } \\
\text { Internet y condiciones de otorgamiento de los equipos }\end{array}$ & $\begin{array}{l}\text { Tipo de equipo y sistema operativo } \\
\text { Condiciones de otorgamiento de equipos } \\
\text { a docentes } \\
\text { Condiciones de otorgamiento de equipo a } \\
\text { estudiantes }\end{array}$ \\
\hline $\begin{array}{l}\text { Percepciones de las } \\
\text { docentes en relación } \\
\text { con el uso de las } \\
\text { portátiles }\end{array}$ & $\begin{array}{l}\text { Son el conjunto de ideas o creencias que las docentes } \\
\text { participantes del estudio tienen en relación con: capa- } \\
\text { citación mantenimiento e infraestructura del proyecto; } \\
\text { participación estudiante-docente-comunidad; utiliza- } \\
\text { ción del equipo en procesos de enseñanza y aprendizaje: } \\
\text { ubicuidad y uso del equipo por parte de estudiantes }\end{array}$ & $\begin{array}{l}\text { Positivo: porcentaje de respuestas positivas } \\
\text { Negativo: porcentaje de respuestas } \\
\text { negativas }\end{array}$ \\
\hline
\end{tabular}

Fuente: elaboración propia con base en el marco conceptual del proyecto.

\section{RESULTADOS}

A continuación, se exponen los resultados de la investigación en función de la percepción docente sobre su participación en las instancias que llevaban a cabo experiencias del modelo «1 a 1». Las iniciativas participantes presentan como condición haber entregado equipos a escuelas del I y II Ciclo de Educación General Básica. Los resultados se exponen de forma total en ambas iniciativas.

Las docentes del sexo femenino conforman 70,2\% (33) de la muestra y $29,8 \%$ (14) el masculino. Las personas participantes poseen un alto nivel académico, 53,2\% (25) posee una maestría, 44,7\% (21) licenciatura y $2,1 \%$ (1) el grado de diplomado (Tabla 2 )

En cuanto a la tenencia de dispositivos móviles: $100 \%$ (47) cuenta con un teléfono celular y una computadora portátil, $51,1 \%$ (24) con una cámara de fotos digital, 34\% (16) con una tableta y $23,4 \%$ (11) con un reproductor de música digital.

\section{Contexto educativo donde se desenvuelve}

De acuerdo con la información suministrada, entre los docentes de Fundación Omar Dengo y Fundación Quirós Tanzi, 29\% (14) se desempeña como asesor de informática educativa, los demás laboran en instituciones de educación pública, dirección 1 25,5\% (12), dirección 2 21,3\% (10), dirección 3 4,3\% (2) y unidocente $12,8 \%$ (6) y privada $6,4 \%$ (3). 
De los docentes entrevistados 53,2\% (25) labora en diferentes iniciativas: para el proyecto Conectándonos MEP-FQT, 40,4\% (19) para la Fundación Omar Dengo-PRONIE, y 6,4\% (3) en otras, es posible que estas tres personas no estuvieran seguras de la iniciativa en la que participaban al momento de contestar el instrumento.

TABLA 2

Condiciones de infraestructura con que cuenta la institución para hacer uso del recurso tecnológico

\begin{tabular}{lcc} 
& $\boldsymbol{n}$ & $\%$ \\
\hline Buenas & 17 & 36,2 \\
\hline Malas & 3 & 6,4 \\
Muy buenas & 10 & 2,3 \\
\hline Muy malas & 2 & 4,3 \\
\hline Regulares & 15 & 3,9
\end{tabular}

Fuente: elaboración propia con base en el instrumento.

Las condiciones de infraestructura con que cuenta la institución para utilizar el recurso tecnológico son las siguiente: $36,2 \%$ (17) buenas, $21,3 \%$ (10) muy buenas, 31,9 (15) regulares, $6,4 \%$ (3) malas y $4,3 \%(2)$ muy malas.

TABLA 3

Condición de asignación de equipos portátiles al docente dentro de la iniciativa

\begin{tabular}{|c|c|c|}
\hline & $n$ & $\%$ \\
\hline $\begin{array}{l}\text { Cuento con una computadora portátil que utilizo a título personal y me fue otorgada } \\
\text { por la iniciativa en la que estoy participando }\end{array}$ & 18 & 54,4 \\
\hline Cuento con una computadora portátil que adquirí por mis propios medios & 6 & 18 \\
\hline Cuento con una computadora portátil que utilizo a título personal y me dio la escuela donde trabajo & 3 & 9 \\
\hline Otra condición no especificada (no sabe) & 3 & 9 \\
\hline No cuento con una computadora portátil en la institución & 2 & 6 \\
\hline Cuento con acceso a una computadora portátil que compartimos con varios docentes & 1 & 3 \\
\hline
\end{tabular}

Fuente: elaboración propia con base en el instrumento.

* De esta categoría se excluyó a 14 asesores de la iniciativa FOD-PRONIE debido a su función es asignar los equipos.

Indicó que cuenta con una computadora portátil 54,4 \% (18) de las personas que la utiliza a título personal y que le fue otorgada por la iniciativa en la que participa, mientras $18 \%$ (6) señaló que cuenta con una laptop que adquirió por sus propios medios y $9 \%$ (3) señaló que cuenta con una laptop que utiliza a título personal y se la facilitó la escuela donde trabaja, también indicaron que posee una laptop propia y una de las XO como la de los niños, pero que utilizan una computadora personal ya que la XO es muy pequeña y no reúne los requisitos para el docente. Por otra parte, $6 \%$ (2) no cuenta con una laptop en la institución y $3 \%$ (1) solo cuenta con acceso a una computadora que comparte con varios docentes. 
TABLA 4

Sistema operativo con que cuenta el equipo portátil asignado a docentes

\begin{tabular}{lcc|} 
& $\boldsymbol{n}$ & $\%$ \\
\hline Windows & 33 & 70,2 \\
\hline Linux Sugar & 11 & 23,4 \\
No sabe & 3 & 6,4
\end{tabular}

Fuente: elaboración propia con base en el instrumento.

En cuanto al sistema operativo, 70,2\% (33) utiliza Windows, 23,4\% (11) posee Linux Sugar, 4,3\% (2) no sabe cuál es y 2,1\% (1) señaló que cuenta con otro tipo (Tabla 5).

Sobre el equipo asignado al estudiantado $85,1 \%$ (40) cuenta con una laptop que le fue otorgada por la iniciativa en la que está inscrito, mientras $8,5 \%$ (4) cuenta con una laptop entregada por la escuela y $6,4 \%$ (3) debe financiársela.

TABLA 5

Sistema operativo con que cuenta el equipo asignado a estudiantes

\begin{tabular}{lcc|} 
& $\boldsymbol{n}$ & $\%$ \\
\hline Windows & 24 & 51,1 \\
\hline Linux Sugar & 19 & 40,4 \\
No sabe & 4 & 8,5 \\
\hline
\end{tabular}

Fuente: elaboración propia con base en el instrumento.

En cuanto al sistema operativo 51,1\% (24) utiliza Windows, 40,4\% (19) posee Linux Sugar, 8,5\% (4) señaló que no sabe. Referente al permiso para llevar las portátiles a sus hogares, 95,7\% si cuenta con el permiso, frente a $4,3 \%$ que no (2). Dentro de las razones que se consideran para dar el permiso de que se lleven los equipos a sus casas se indicaron:

TABLA 6

Detalle de la condición de asignación a equipo a estudiantes de acuerdo con los participantes del estudio

Así está implícito en el convenio entre la FQT y los padres de familia

Depende de la propuesta sea Multigrado, REMA, Movilab, Indígena o Laboratorio.

Es parte de la familiarización con el recurso y para desarrollar temas extraclase.

Los padres de familia en esta modalidad firman un contrato en donde se hacen responsables de que sus hijos se las lleven al hogar.

En el caso del modelo «1 a 1», los estudiantes se llevan las máquinas a sus casas, debido a que los papás o responsables firmaron cartas de compromiso para cuidarlas y utilizarlas en lo pedagógico.

En los proyectos «1 a 1» si se la pueden llevar; en la modalidad laboratorio no.

Es un convenio con la FOD.

La llevan a sus casas.

Los estudiantes las llevan al hogar y deben traerlas todos los días a la escuela ya que se utilizan como herramienta durante las lecciones.

Según contrato del padre de familia con la FQT

Fuente: elaboración propia con base en el instrumento. 


\section{Percepciones docentes sobre las iniciativas en que participan}

Cómo parte de los elementos del trabajo se indagó con los docentes la percepción con respecto a elementos de manteamiento, infraestructura, participación, uso de las computadoras en los procesos de enseñanza y aprendizaje, ubicuidad y autonomía en el uso de los equipos. Las anteriores variables se consideran en función de los principios del modelo «1 a 1» que se explicaron inicialmente. A continuación, se expone las percepciones en relación con capacitación, mantenimiento e infraestructura.

- De las instituciones, $68,1 \%$ de las que otorgó las computadoras portátiles brindó siempre el entrenamiento a los docentes en su uso; $14,9 \%$ indicó que casi siempre o de vez en cuando y $2,1 \%$ que no se brindó nunca.

- De los estudiantes, $46,8 \%$ recibió asesoría para utilizar las computadoras portátiles por parte de la institución que dio los equipos; $19.1 \%$ casi siempre, $17 \%$ de vez en cuando, 10,6\% casi nunca y $6,4 \%$ nunca.

- En el caso de los participantes, 83\% manifiesta haber recibido mantenimiento y soporte de las computadoras por parte de las iniciativas, 8,5\% indicó que la situación se presenta casi siempre o de vez en cuando.

Nunca Casinunca De vez en cuando $\square$ Casi siempre $\square$ Siempre

Se cuenta con acceso internet para el uso de las computadoras portátiles en la institución

La institución que dio los equipos portátiles da mantenimiento y soporte a las computadoras

Los estudiantes recibieron asesoría para aprender a utilizar las computadoras portátiles por parte de la institución que dio los equipos

La institución que otortó las computadoras portátiles les brindó entrenamiento a los docentes en el uso de éstas.

2,1

\section{8,5}

8,5

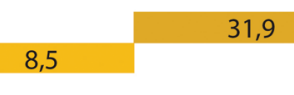

8,5

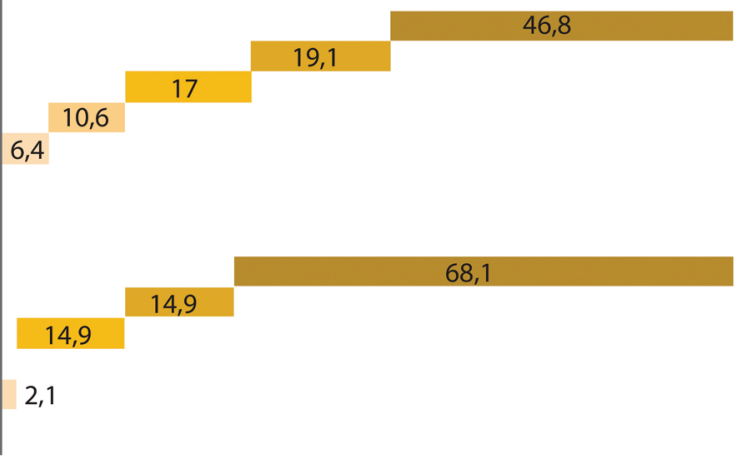

Porcentaje

Figura 1. Capacitación, mantenimiento e infraestructura. Fuente: elaboración propia con base en el instrumento. 


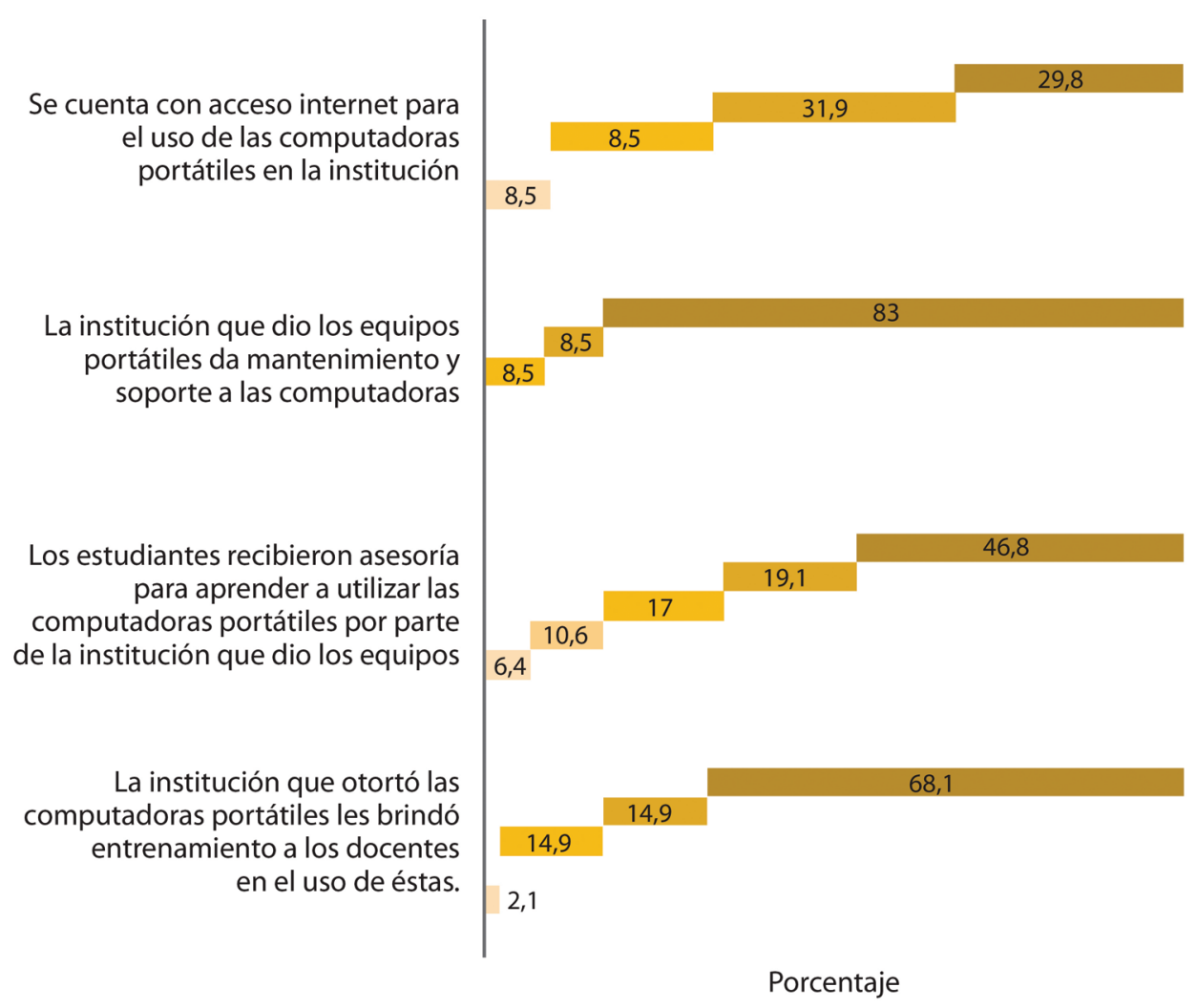

Figura 2. Participación de los actores del proceso educativo. Fuente: elaboración propia con base en el instrumento.

- De las personas participantes, $29,8 \%$ indica contar con acceso a internet para el uso de las computadoras portátiles en su institución, mientras un31,9\% indicó que la institución casi siempre cumple con con el acceso a internet, un 21,3\% indica que lo cumple de vez en cuando y 8,5\% indicó que nunca contar con este recurso.

- Indicó que siempre trabaja con los estudiantes desarrollando actividades de aprendizaje a través del uso de las computadoras portátiles $29,8 \%$ de los participantes, $34,0 \%$ señaló que la situación se presenta casi siempre; $31,9 \%$ de vez en cuando y $2,1 \%$ que casi nunca o nunca se presenta.

- De los docentes, $14,9 \%$ siempre trabaja con otros colegas para desarrollar actividades interdisciplinarias con las computadoras portátiles, $29,8 \%$ indicó que la situación se presenta casi siempre, $40,4 \%$ señaló que se presenta de vez en cuando, $10,6 \%$ casi nunca y $4,3 \%$ expresó que nunca se dio.

- De los docentes, 46,8\% señaló que los estudiantes siempre participan activamente en las actividades propuestas que requieren trabajar con las computadoras portátiles, $44,7 \%$ indicó casi siempre y $8,5 \%$ de vez en cuando.

- $\quad$ El porcentaje de docentes que señaló que los padres de familia participan siempre en los trabajos desarrollados por sus hijos mediante la computadora fue de $4,3 \%$, mientras $21,3 \%$ indicó que casi siempre, $53,2 \%$ de vez en cuando; $14,9 \%$ casi nunca y para $6,4 \%$ nunca. 
En lo referente al uso las computadoras en los procesos de enseñanza-aprendizaje, los docentes señalaron:

- De la muestra, 55,3\% indicó que siempre desarrolla estrategias educativas a través de las computadoras portátiles, lo cual proporciona una oportunidad para mejorar el proceso de aprendizaje; $34,0 \%$ señaló que la situación se presenta casi siempre y para 10,6\% de vez en cuando.

- De los docentes, $68,3 \%$ considera que utilizar la computadora como una herramienta de apoyo para el aprendizaje aumenta el interés del estudiante por las temáticas de estudio, 25,5\% que la situación se presenta casi siempre y para $6,4 \%$ de vez en cuando.

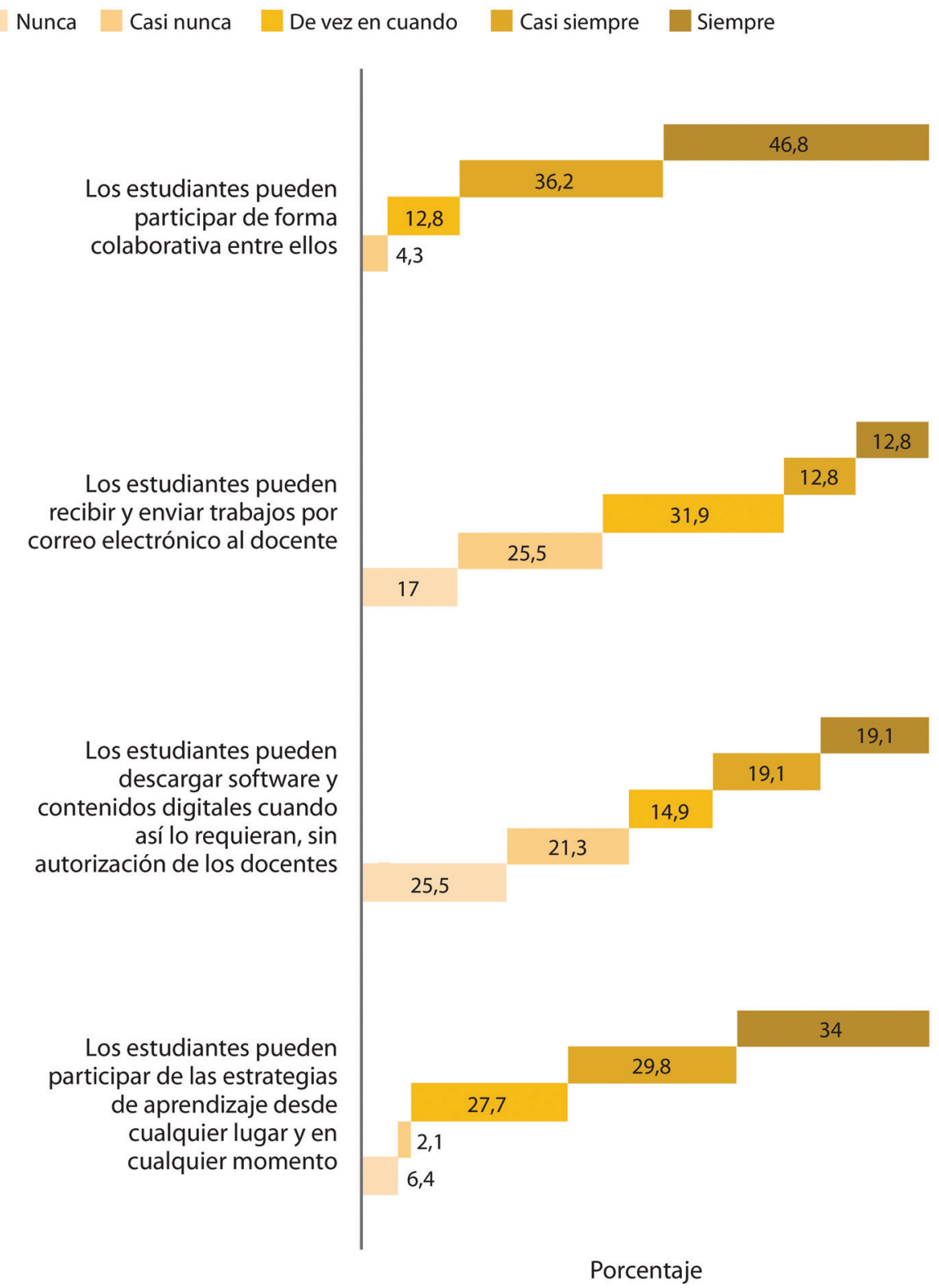

Figura 3. Ubicuidad y autonomía en el uso de equipos portátiles.

Fuente: elaboración propia con base en el instrumento. 
- De los docentes, 55,3\% expresó que los estudiantes con dificultades de aprendizaje pueden beneficiarse de las posibilidades didácticas que implica el uso de la computadora; 34,0\% indicó que la situación se presenta casi siempre y $8,5 \%$ que se presenta de vez en cuando, mientras $2,1 \%$ que casi nunca se presentó.

- De los docentes, 44,7\% señaló que el desarrollo de estrategias educativas con el uso de las computadoras siempre puede ayudarle a personalizar el aprendizaje de los estudiantes; $46,8 \%$ indicó que casi siempre y $8,5 \%$ que de vez en cuando.

- Señalaron que el empleo de la computadora como herramienta de apoyo ha mejorado su eficacia en el planteamiento de estrategias educativas $38,3 \%$ de los docentes, mientras $46,8 \%$ indicó que casi siempre y $14,9 \%$ contestó que de vez en cuando.

- De los docentes, $46,8 \%$ ha tenido más experiencias positivas que negativas con el uso de las computadoras como apoyo en el proceso de enseñanza; $36,2 \%$ indicó que casi siempre y $17 \%$ de vez en cuando.

- De la muestra, 34,0\% indicó que los estudiantes siempre pueden participar de las estrategias de aprendizaje desde cualquier lugar y en cualquier momento; $29,8 \%$ que la situación se presenta casi siempre; $27,7 \%$ de vez en cuando; $2,1 \%$ casi nunca y para $6,4 \%$ no se presenta la situación.

- De los docentes, 19,1\% considera que los estudiantes siempre o casi siempre pueden descargar software y contenidos digitales cuando así lo requieran, sin autorización de los docentes; $14,9 \%$ indicó que la situación se presenta de vez en cuando, $21,3 \%$ casi nunca y $25,5 \%$ señaló que nunca.

- De la muestra, $12,8 \%$ señaló que los estudiantes siempre o casi siempre pueden recibir y enviar trabajos por correo electrónico al docente; 31,9\% indicó que la situación se presenta de vez en cuando, $25,5 \%$ indicó que casi nunca y para $17 \%$ nunca.

- De los docentes, $46,8 \%$ señaló que los estudiantes siempre pueden participar de forma colaborativa entre ellos; $36,2 \%$ indicó que la institución casi siempre cumple con esta situación, 12,8\% la cumple de vez en cuando y $4,3 \%$ casi nunca.

En cuanto al hecho de que si el docente ha recibido asesoría en el uso de computadoras portátiles en los últimos seis meses $74,5 \%$ (35) indicó que sí la han recibido y $25,5 \%$ (12) que no.

\section{DISCUSIÓN Y CONCLUSIONES}

En Costa Rica se han desarrollado durante varios años iniciativas «1 a 1». Antes del modelo más reconocido en el ámbito internacional, One Laptop per Child, ya en el país se aplicaba y experimentaba con equipos portátiles en las aulas. Las iniciativas nacionales del modelo «1 a 1» que fueron identificadas se llevaron a cabo bajo el acompañamiento de una organización, se reconocen la Fundación Omar Dengo, la Fundación Quirós-Tanzi, e Intel Educar como las principales.

A nivel de política educativa, las acciones se dieron bajo un esquema planificado para la asignación de equipos, en todos los casos siempre con algún acompañamiento estatal por parte del MEP. Lo anterior contrasta con otras situaciones y contextos internacionales, donde los equipos han sido otorgados bajo un esquema "caritativo" o con un tinte político asistencialista (Selwyn, 2013).

En cuanto a contexto educativo donde se desenvuelven las iniciativas, el personal docente que participa en experiencias con tecnologías móviles del tipo «1 a 1», posee un nivel académico que oscila entre el 
grado de licenciatura y maestría, por lo que se considera que posee las condiciones y competencias profesionales necesarias para la orientación del estudiantado en el proceso de enseñanza aprendizaje bajo este modelo. Sin embargo, la capacitación especializada al cuerpo docente es fundamental. El asesorar de forma permanente marca la diferencia en los usos pedagógicos que las y los docentes propongan realizar a los estudiantes con estos equipos (Zheng, Warschauer, Lin, y Chang, 2016).

En relación con el uso de equipos, existen restricciones en algunos aspectos, como descarga de software; no obstante, hay libertad para llevar los equipos a las casas.

Por otra parte, no hay claridad sobre el grado de involucramiento de la comunidad y la familia en las actividades con portátiles. Se requiere profundizar sobre este aspecto en otras investigaciones, no obstante, también es necesario una mayor capacitación a los padres de familia para que puedan interactuar con sus hijos cuando realizan trabajos con el computador.

También, aunque hay una asesoría inicial por parte de las iniciativas a los docentes para el manejo del computador y el software que contiene, resulta vital que las asesorías se mantengan con el tiempo y no solo sean iniciales (Iriarte Diazgranados, Domínguez Merlano, y Ricardo Barreto, 2013).

Uno de los aspectos que evaluaciones recientes enfatizan es la necesidad de mejora en la infraestructura y la capacitación (Steve Vosloo, 2014), lo que coincide con los hallazgos del estudio. La infraestructura es uno de los aspectos más importantes que señalan los participantes. Existen carencias, en especial en acceso a Internet de alta velocidad, que permitan explotar más el trabajo que se puede desarrollar con los equipos, de acuerdo con la gama de posibilidades que se pueden desarrollar con el software y los recursos en línea. Cabe destacar que las limitaciones de acceso a Internet se han convertido en una nueva brecha digital (Riddlesden y Singleton, 2014) es decir iniciativas que surgen para disminuir una brecha, evidencia nuevas brechas tecnológicas.

Cabe señalar que en algunas de las iniciativas existe un vínculo de apoyo y asesoría a los docentes, antes y durante el proceso, las cuales representan los casos de mayor aprovechamiento del equipo. En aquellas donde no existe suficiente capacitación se promueven la subutilización de los equipos. Los profesores requieren de más técnicas pedagógicas e inducción para implementarlas en el aula con apoyo de las TIC. Lo cual es contradictorio, y habría que valorar, en contraposición con lo que autores como Castro (2014) expresan, que sea el mismo docente su propio y único gestor de los conocimientos en el uso de portátiles en el aula.

El mantenimiento constante del equipo computacional resulta vital y necesario para solucionar los problemas de mal funcionamiento comunes que se dan en los equipos debido a su uso constante.

En cuanto a la participación de los actores en el proceso educativo, resulta fundamental que los profesores sean dotados de equipo semejante al que poseen los estudiantes con el fin de que puedan explorar el sistema operativo y el software instalados en el computador. Así como las limitaciones de espacio en disco duro y memoria, con el fin de poder plantear actividades que puedan correr en los equipos que poseen los estudiantes.

Es imprescindible que tanto estudiantes como docentes cuenten con el equipo suficiente y necesario, así como con el mantenimiento permanente por parte de las entidades encargadas de los proyectos, con el fin de facilitar el ejercicio de la práctica docente y poder obtener el mayor aprovechamiento de las actividades de enseñanza aprendizaje propuestas. Esto coincide con los estudios de política comparativa, donde aún se manifiesta que es un reto el cómo integrar esta tecnología a las aulas, en los planes de estudio y en el currículo (UNESCO, 2016). En este sentido también se apoya la idea de generar guías para el abordaje de contenidos y habilidades curriculares necesarias. 
En cuanto a ubicuidad y la autonomía en el uso de equipos portátiles, los estudiantes aún utilizan poco el equipo para intercambiar correos electrónicos relacionados con trabajos académicos, así como descargar contenidos específicos ya que muchos equipos sufren de limitaciones de descarga.

Finalmente, señalar que del estudio se desprenden nuevos desafíos de investigación. Uno es llevar a cabo una investigación de mayor escala, con una estrategia metodológica experimental o cuasi experimental, que permita llegar a conclusiones más definitivas sobre lo que sucede en las aulas con los modelos «1 a 1». Así como profundizar mediante investigaciones cualitativas en las estrategias pedagógicas que ya son utilizadas y su efectividad, no muchos estudios han podido dar cuenta de la adquisición de los aprendizajes por medio de herramientas tecnológicas (Zheng et al., 2016).

En conclusión, conforme la tecnología se hace cada día menos costosa, y más común en las aulas, se crean nuevos escenarios educativos para estudiantes y docentes pero se evidencian nuevas brechas tecnológicas, se reafirma la importancia de la asesoría y la capacitación permanente y se plantean nuevos desafíos para la investigación.

\section{REFERENCIAS}

Alvarado-Barrantes, E. (2007). Experiencias en estrategias pedagógicas con el uso de la tecnología. Revista Electrónica Educare, Ext(2), 253-256. Recuperado a partir de http://www.revistas.una.ac.cr/ index.php/EDUCARE/article/view/1400

Area, M. (2011). Los efectos del modelo 1:1 en el cambio educativo en las escuelas. Evidencias y desafíos para las políticas iberoamericanas. Revista Iberoamericana de Educación, 56, 49-74. Recuperado de http://www.rieoei.org/rie56a02.pdf

Castro, H. (2014). Proceso de gestión del Proyecto de Tecnologías Móviles: Estudio de caso. Gestión de la Educación, 4(1), 103-137. Recuperado a partir de https://revistas.ucr.ac.cr/index.php/gestedu/ article/download/12921/12216

Claro, M., Nussbaum, M., López, X., \& Díaz, A. (2013). Introducing 1 to 1 in the classroom: A large-scale experience in Chile. Educational Technology y Society, 16(3), 315-328.

Davies, R. S., \& West, R. E. (2014). Technology Integration in Schools. En J. M. Spector, M. D. Merrill, J. Elen, y M. J. Bishop (Eds.), Handbook of Research on Educational Communications and Technology (Vol. 13, pp. 841-853). New York, NY: Springer New York. http://doi.org/10.1007/978-1-4614-3185-5_68

Day-Ashley, L. (2012). Case Study Research. En J. Arthur, M. Waring, R. Coe, y L. V. Hedges (Eds.), Research Methods and Methodologies in Education (pp. 104-107). London: SAGE Publications Ltd.

Diodato, M. (2007). Innovative Age: Technology for Education in the Developing World. Harvard International Review, 28(4), 38-41.

Edelman, A., y Fernández, A. (2010). Plan Ceibal «One Laptop Per Child» en Uruguay. Revista de Antiguos Alumnos Del IEEM, 13(1), 24-50. Recuperado de http://dialnet.unirioja.es/servlet/ articulo?codigo $=3164162$ yorden $=343889$ yinfo $=$ lin

Fallas, I. y Zúñiga, M. (2011). Estado del Arte de las Tecnologías Digitales de la Información y la Comunicación en la Educación Costarricense. Ponencia para el Tercer Informe Estado de la Educación. San José, Costa Rica: CONARE. Disponible en: http://www.estadonacion.or.cr/files/biblioteca_virtual/educacion/003/Fallas_Zuniga_2010_TIC_Educacion.pdf

Flyvbjerg, B. (2011). Case Study. En N. K. Denzin y Y. S. Lincoln (Eds.), The Sage Handbook of Qualitative Research (4. ${ }^{a}$ ed., pp. 301-316). SAGE Publications, Ltd

Fundación OmarDengo.(s.f.). Informática Educativa (PRONIEMEP-FOD). Recuperado 1 deoctubrede 2016, a partir de http://www.fod.ac.cr/index.php?option=com_contentyview=articleyid=81yltemid=160 
Fundación Quirós Tanzi. (2011). Principios: Nuestros 5 pilares. Recuperado de http://fundacionqt.org/principios.php

Fundación Quirós Tanzi. (2013). Memoria 2013. San José, Costa Rica. Recuperado a partir de http://fundacionqt.org/

Hernández Sampieri, R., Fernández Collado, C., \& Baptista Lucio, P. (2010). Metodología de la investigación (5. ed.). Lima, Perú.: McGraw-Hill.

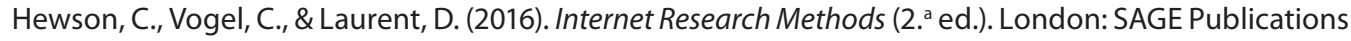
Ltd.

Holcomb, L. (2009). Results y Lessons Learned from1:1 Laptop Initiatives: A Collective Review. TechTrends, 53(6), 49-55. http://doi.org/10.1007/s11528-009-0343-1

INEC. (2011).X Censo Nacional de Población y VI de Vivienda 2011: Resultados Generales. San José, Costa Rica.

Iriarte Diazgranados, F., Domínguez Merlano, E., \& Ricardo Barreto, C. (2013). Resultados del piloto de innovación 1 a 1 en las prácticas pedagógicas de los docentes. (Spanish). Results of the Innovation 1 to 1 Pilot Project in Teacher's Pedagogical Practices. (English), (38), 27-39. Recuperado a partir de http:// search.ebscohost.com/login.aspx?direct=trueydb=fuayAN=90648363ylang=esysite=ehost-live

Kozma, R. B., \& Vota, W. S. (2014). ICT in Developing Countries: Policies, Implementation, and Impact. En J. M. Spector, M. D. Merrill, J. Elen, y M. J. Bishop (Eds.), Handbook of Research on Educational Communications and Technology (pp. 885-894). New York, NY: Springer New York. doi:10.1007/978-1-4614-3185-5

MEP. (2014). «La Educación Subversiva: Atreverse a construir el país que queremos» Memoria Institucional MEP 2006 - 2014. San José, Costa Rica. Recuperado de http://www.mep.go.cr/sites/default/files/ page/adjuntos/memoriainstitucional20062014pdf.pdf

MEP. (2016).Programa Nacional de Tecnologías Móviles para la Educación Tecno@aprender. Conexiones: una experiencia más allá del aula, 8(3). Recuperado a partir de http://www.mep.go.cr/educatico

Nugroho, D., \& Londsale, M. (2010). Evaluation of OLPC programs globally: a literature review. Melbourne, Australia: Australian Council for Educational Research. Recuperado de http://wiki.laptop.org/images/a/a5/OLPC_Lit_Review_v4_Aug2010.pdf

One Laptop per child (s.f.). Worldwide over 2.4 million children and teachers have xo laptops. Recuperado de: http://one.laptop.org/map

One Laptop per Child Project. (2011). Costa Rica se está preparando para lanzar una laptop por niño en 2012. Recuperado de http://www.olpcnews.com/countries/costa_rica/costa_rica_se_esta_preparando_la_olpc.html

Pachler, N., Bachmair, B., \& Cook, J. (2010). The Mobile Complex, Socialization and Learning Resources. En Mobile Learning (pp. 205-222). Boston, MA:Springer US. http://doi.org/10.1007/978-1-4419-0585-7_8

Penuel, W. (2006). Implementation and effects of one-to-one computing initiatives: A research synthesis. Journal of Research on Technology in Education, 5191, 329-348. Recuperado de http://www.tandfonline.com/doi/abs/10.1080/15391523.2006.10782463

Proyecto Estado de la Nación. (2011). Tercer Informe del Estado de la Educación. San José, Costa Rica. Recuperado de http://www.estadonacion.or.cr/

Riddlesden, D., \& Singleton, A. D. (2014). Broadband speed equity: A new digital divide? Applied Geography, 52, 25-33. http://doi.org/10.1016/j.apgeog.2014.04.008

Ryan, Z. (2014). One Laptop per Child (OLPC) XO Laptop. Art Institute of Chicago Museum Studies, 35(2), 28-29. Recuperado a partir de http://www.jstor.org/stable/40652397 
Selwyn, N. (2013). «Empowering the World's Poorest Children»? A Critical Examination of One Laptop per Child. En The Politics of Education and Technology (pp. 101-125). New York: Palgrave Macmillan US. http://doi.org/10.1057/9781137031983_6

Severin, E., y Capota, C. (2011). Modelos uno a uno en América Latina y el Caribe: Panorama y perspectivas. Banco Interamericano de Desarrollo. Recuperado de http://publications.iadb.org/ handle/11319/5485

Vosloo, S. (2014). 1:1 Educational Computing Initiatives - Lessons learned and confirmed. En Global Symposium on ICT in Education 2014. Gyeongju, Republic of Korea.

The OLPC Wiki. (s.f.). Los Cinco Principios de la OLPC. Recuperado 15 de octubre de 2016, a partir de http://wiki.laptop.org/go/Core_principles/lang-es

Tymms, P. (2012). Questionnaires. En J. Arthur, M. Waring, R. Coe, \& L. V. Hedges (Eds.), Research Methods and Methodologies in Education (pp. 231-240). London: SAGE Publications Ltd.

UNESCO. (2016). Revisión comparativa de iniciativas nacionales de aprendizaje móvil en América Latina:Los casos de Colombia, Costa Rica, Perú y Uruguay. París, Francia. Recuperado a partir de http://unesdoc. unesco.org/images/0024/002439/243976s.pdf

Vacchieri, A. (2013). Estado del arte sobre la gestión de las políticas de integración de computadoras y dispositivos móviles en los sistemas educativos. Buenos Aires, Argentina: UNICEF.

Valiente, O. (2010). 1-1 in Education: Current Practice, International Comparative Research Evidence and Policy Implications (No. 44). doi:10.1787/19939019

Warschauer, M., \& Ames, M. (2010). Can One Laptop per Child save the world's poor? Journal of International Affairs, 64(1), 33-51. Recuperado de

Zheng, B., Warschauer, M., Lin, C.-H., \& Chang, C. (2016). Learning in One-to-One Laptop Environments: A Meta-Analysis and Research Synthesis. Review of Educational Research, 86(4), 1052-1084. http:// doi.org/10.3102/0034654316628645

Zucker, A. A., \& Light, D. (2009). Laptop programs for students. Science, 323(5910), 82-5. doi:10.1126/ science. 1167705 